\title{
Sensing limitations in the Lion and Man problem
}

\author{
Shaunak D. Bopardikar
}

\author{
Francesco Bullo
}

\author{
João P. Hespanha
}

\begin{abstract}
We address the discrete-time Lion and Man problem in a bounded, convex, planar environment in which both players have identical sensing ranges, restricted to closed discs about their current locations. The evader is randomly located inside the environment and moves only when detected. The players can step inside identical closed discs, centered at their respective positions. We propose a sweep-pursuit-capture strategy for the pursuer to capture the evader. The sweep phase is a search operation by the pursuer to detect an evader within its sensing radius. In the pursuit phase, the pursuer employs a greedy strategy of moving towards the last-sensed evader position. We show that in finite time, the problem reduces to a previously-studied problem with unlimited sensing, which allows us to use the established Lion strategy in the capture phase. We give a novel upper bound on the time required for the pursuit phase to terminate using the greedy strategy and a sufficient condition for this strategy to work in terms of the value of the ratio of sensing to stepping radius of the players.
\end{abstract}

\section{INTRODUCTION}

The classical Lion-Man problem is a game posed as to determine a strategy for a pursuer(lion) to capture an evader(man) in a given environment. By capture, we mean that the evader and the pursuer at the same position after a finite time. The aim of the pursuer is to capture the evader for any evader trajectory. The evader wins the game if it can avoid capture indefinitely. Both the players have identical motion capabilities. Capture strategies are important in surveillance where we would like to detect and capture equally agile intruders. Another application is search-andrescue operations where a worst-case capture strategy guarantees a rescue, in spite of any unpredictable motion of the victim.

\section{A. Contributions}

We address the case of limited sensing capability: the pursuer and the evader can sense each other's position only if the distance between them is less than or equal to a given sensing radius. The motion of both players is restricted to closed discs of given stepping radius, centered at their respective current positions. The game is played in a bounded, convex, planar environment which is assumed to be known to both players. The evader is at an arbitrary location inside the environment, at the start of the game. It follows a reactive rabbit model, i.e., does not move until it senses a pursuer [1]. The pursuer sweeps the environment in a definite path until

\footnotetext{
Shaunak D. Bopardikar and Francesco Bullo are with the Department of Mechanical Engineering, University of California at Santa Barbara, Santa Barbara, CA 93106, USA, \{shaunak, bullo\}@engineering.ucsb.edu

João P. Hespanha is with the Department of Electrical and Computer Engineering, University of California at Santa Barbara, Santa Barbara, CA 93106, USA, hespanha@ece.ucsb.edu
}

the evader is sensed, which must necessarily happen in finite time. We then establish how a greedy strategy of moving to the last-sensed location of the evader, reduces the problem to the previously-studied problem with unlimited sensing. Our analysis gives a novel upper bound on the time required for the pursuit phase to terminate, which is not known so far in literature. Further, we give a sufficient condition on the value of the ratio of sensing to stepping radius $\kappa$ of the players, so that capture takes place. We also give a sufficient condition on the environment so that there exists an evasion policy against the greedy pursuer strategy. We then use the established Lion strategy [5] to complete the capture.

\section{B. Related Work}

The continuous time version of this problem has been studied by Ho et al. [2], Lim et al. [3] and Pachter [4] to cite a few. Recently, significant attention has been received by the discrete-time version of the game. The paper by Sgall [5] gives sufficient conditions and the Lion strategy for a single pursuer to capture an evader in a semi-open environment. This strategy has been extended to multiple pursuers in an unbounded environment by Kopparty and Ravishankar [6]. Recently, Alexander et al. [7] proposed a simple greedy strategy in which the pursuer moves towards the last position of the evader and characterized environments in which the strategy is guaranteed to work. But no upper bound on the time taken is known. The game has also been studied in different types of bounded environments, e.g., circular environment by Alonso et al. [8], curved environments by LaValle et al. [9]. Visibility-based pursuit evasion has been studied by Guibas et al. [10], Sachs et al. [11] and in polygonal environments by Isler et al. [12].

Each of above mentioned works proposes strategies which require that the pursuers have unlimited sensing capacity. In this context, Gerkey et al. [13] have studied a version of visibility limited to an angle, instead of the entire region. Isler et al. [1] have considered the problem on a graph, with the visibility limited to nodes adjacent to the current node of a player. A framework which uses probabilistic models for sensing devices for the agents can be found in the works of Hespanha et al. [14] and Vidal et al. [15].

\section{Organization}

The problem formulation is described in section II. The pursuer strategy is described using three phases given in sections III-A, III-B and III-C, with section III-A describing the sweep strategy, section III-B discussing the aspects of the greedy strategy and finally section III-C showing the application of the Lion strategy [5] for completing capture. 


\section{Problem Set-uP}

We assume that initially the evader is arbitrarily located inside a bounded, convex, planar environment, $\mathcal{Q} \subset \mathbb{R}^{2}$. We assume a discrete-time model with alternate motion of the evader and the pursuer. Both players know the entire environment. Define $\mathcal{Q}_{\mathrm{e}}=\mathcal{Q} \cup \phi$, where $\mathcal{Q}$ is the environment and $\phi$ is the null element. Here, the null element refers to the fact that during sensing, the measurement of the position of an evader may not be available to the pursuer. Let $e[t]$ and $p[t]$ denote the absolute positions of the evader and the pursuer respectively, at time $t$. The equations of motion, in discrete-time, can be written as,

$$
\begin{aligned}
& e[t+1]=e[t]+u^{e}\left(e[t], y^{p}[t]\right), \\
& p[t+1]=p[t]+u^{p}\left(e[t], y^{e}[t+1], p[t]\right),
\end{aligned}
$$

where $y^{p}[t] \in \mathcal{Q}_{\mathrm{e}}$ is the measurement of the pursuer position taken by the evader at the $t^{t h}$ time instant and $y^{e}[t+1] \in \mathcal{Q}_{\mathrm{e}}$ is the measurement of the evader position taken by the pursuer at the $(t+1)^{t h}$ time instant. We assume that the players can sense each other only if the distance between them is less than or equal to the sensing radius $r_{\text {sens. }}$ Thus,

$$
y^{p}[t]= \begin{cases}p[t], & \text { if }\|p[t]-e[t]\| \leq r_{\text {sens }} \\ \phi, & \text { otherwise. }\end{cases}
$$

Similarly,

$$
y^{e}[t+1]= \begin{cases}e[t+1], & \text { if }\|p[t]-e[t+1]\| \leq r_{\text {sens }}, \\ \phi, & \text { otherwise. }\end{cases}
$$

The functions $u^{e}: \mathcal{Q}_{\mathrm{e}} \times \mathcal{Q} \rightarrow \mathcal{Q}$ and $u^{p}: \mathcal{Q}_{\mathrm{e}} \times \mathcal{Q}_{\mathrm{e}} \times$ $\mathcal{Q} \rightarrow \mathcal{Q}$ are termed as strategies for the evader and pursuer respectively. We assume that both players can move with a maximum step size of $r_{\text {step. }}$ This gives,

$$
\left\|u^{e}\right\|,\left\|u^{p}\right\| \leq r_{\text {step }}
$$

The sensing radius $r_{\text {sens }}$ is $\kappa$ times the motion radius $r_{\text {step. }}$ We assume $\kappa$ is greater than 1, i.e., both players can sense further than they can move. The reactive rabbit model for the evader gives $u^{e}=0$ until it is detected. Once detected, the problem is to determine $u^{p}$ that guarantees capture for any evader strategy, $u^{e}$.

\section{The Sweep-Pursuit-CAPture Strategy}

We establish sufficient conditions on the parameter $\kappa$ so that the pursuer can capture the evader. We formally define two notions of capture as follows.

Definition III.1 (Capture notions) The pursuer is said to have captured the evader if after finite time, independent of any evader policy $u^{e}$, the pursuer is at the same position as the evader.

Similarly, the pursuer is said to have trapped the evader within its sensing radius if after a finite time, independent of any evader policy $u^{e}$, the motion circle of the evader is completely contained within the sensing circle of the pursuer.

We now describe the sweep-pursuit-capture strategy.

\section{A. The Sweep phase}

Let $\operatorname{diam}(\mathcal{Q})$ denote the diameter of $\mathcal{Q}$. The pursuer moves with maximum step size along a path, as shown in Figure 1(a) such that the union of the sensing discs of the pursuer at the end of each step contains $\mathcal{Q}$. We term such a path as a sweeping path for $\mathcal{Q}$. We now determine an upper bound on the number of steps for any sweeping path. Consider placing $\mathcal{Q}$ inside a square region of length $\operatorname{diam}(\mathcal{Q})$ and the pursuer moving along a hypothetical sweeping path for the square region, as shown in Figure 1(b). This hypothetical sweeping path is between strips of width $2 r_{\text {step }} \sqrt{\kappa^{2}-\frac{1}{4}}$, parallel to the side. The selection of width for the strips ensures that the pursuer sweeps the entire area of $\mathcal{Q}$, as opposed to if the width were equal to $2 r_{\text {sens }}$, in which case there would be regions which the pursuer would not have detected. There would be $\left\lceil\frac{\operatorname{diam}(\mathcal{Q})}{2 r_{\text {step }} \sqrt{\kappa^{2}-\frac{1}{4}}}\right\rceil$ such strips and it takes at most $\left\lceil\frac{\operatorname{diam}(\mathcal{Q})}{r_{\text {step }}}\right\rceil+\left\lceil\sqrt{\kappa^{2}-\frac{1}{4}}\right\rceil$ steps to sweep one strip completely and be positioned to sweep through a neighboring strip of this hypothetical sweeping path. We obtain the following result.

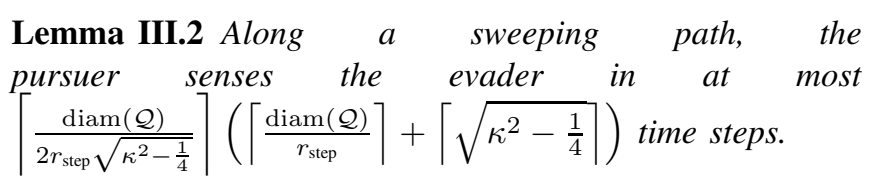

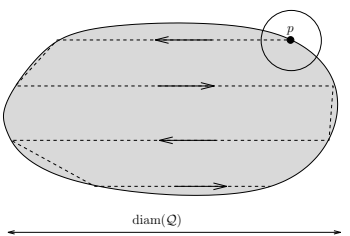

(a) A sweeping path

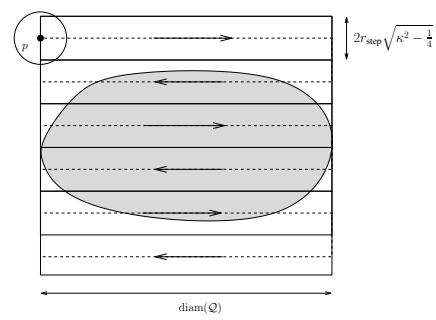

(b) A hypothetical sweeping path
Fig. 1. Sweeping path in single pursuer case

\section{B. The Pursuit phase}

Once the pursuer has sensed the evader (and vice-versa), the evader needs to move in such a way that its new position is not within the current sensing radius of the pursuer. Otherwise the pursuer can move towards the new position of the evader, with maximum step and thus trap it within its sensing disc. We now propose a greedy policy for the pursuer according to which the pursuer must move towards the last sensed position of the evader so as to ensure that it would sense the evader again. It is given by the following control input for the pursuer.

$$
u_{\text {greedy }}^{p}= \begin{cases}r_{\text {step }} \operatorname{vers}\left(y^{e}[t+1]-p[t]\right), & \text { if } y^{e}[t+1] \neq \phi, \\ r_{\text {step }} \operatorname{vers}(e[t]-p[t]), & \text { otherwise. }\end{cases}
$$


where,

$$
\operatorname{vers}(v)= \begin{cases}\frac{v}{\|v\|}, & \text { if } v \neq 0 \\ 0, & \text { if } v=0\end{cases}
$$

After sensing the evader, the trapping time $t^{*}$ is defined as the time taken by the pursuer to trap the evader within its sensing radius. We now present our main result.

Theorem III.3 (Greedy Pursuit) After sensing the evader, the pursuer will trap it within its sensing radius using the greedy strategy if

$$
\kappa \in\left(\sqrt{2+2 \cos \beta_{\mathrm{c}}}, \infty\right),
$$

and the trapping time $t^{*}$ satisfies,

$t^{*} \leq\left(\left\lceil\frac{\log \left(\frac{\kappa-1}{\sqrt{\kappa^{2}-\sin ^{2} \beta_{\mathrm{c}}}-\cos \beta_{\mathrm{c}}-1}\right)}{\log \frac{1}{1-\frac{1-\cos \beta_{\mathrm{c}}}{\kappa}}}\right\rceil+1\right)\left\lceil\frac{\operatorname{diam}(\mathcal{Q})}{\frac{\sqrt{3}}{2} r_{\text {step }}}\right\rceil$,

where

$$
\beta_{\mathrm{c}}=\frac{1}{\left\lceil\frac{\operatorname{diam}(\mathcal{Q})}{\frac{\sqrt{3}}{2} r_{\text {step }}}\right\rceil} \tan ^{-1}\left(\frac{1}{4} \frac{r_{\text {step }}}{\operatorname{diam}(\mathcal{Q})}\right) .
$$

Further, if $\kappa>2$, then as $\frac{\operatorname{diam}(\mathcal{Q})}{r_{\text {step }}} \rightarrow \infty, t^{*} \in$ $O\left(\left(\frac{\operatorname{diam}(\mathcal{Q})}{r_{\text {step }}}\right)^{5}\right)$.

To prove Theorem III.3, we need some preliminary definitions and results which we present now.

\section{Definition III.4 (Deviation and Evasion angles)}

Angle of deviation $\alpha[t] \triangleq \angle e[t+1] p[t+1] e[t]$,

Angle of evasion $\beta[t] \triangleq \alpha[t]+\angle p[t+1] e[t+1] e[t]$,

where the notation $\angle A B C$ refers to the angle between segments $A B$ and $B C$. These angles are illustrated in Figure 2. The following result follows trivially.

Proposition III.5 When the pursuer uses the greedy strategy, for every instant of time $t$,

$$
|\beta[t]| \geq|\alpha[t]|
$$

Note that equality in equation (2) only holds when the evader moves along the line $p[t] e[t]$.

It can be deduced that when the pursuer employs the greedy strategy, the distance between the pursuer and evader is non-increasing. We now define a geometric construction which would be useful in the proof.

Definition III.6 (Cone) $A$ sequence of cones $\mathcal{C}_{k}$ for $k \in$ $\mathbb{Z}_{\geq 0}$ are defined as follows:

(i) Let $t_{0}$ denote the time at the end of the sweep phase. Define cone $\mathcal{C}_{0}$ with $p\left[t_{0}\right]$ as its vertex and the line $p\left[t_{0}\right] e\left[t_{0}\right]$ as the angle bisector, extended to meet the boundary of $\mathcal{Q}$ at point $X$ as shown in Figure 3. Let

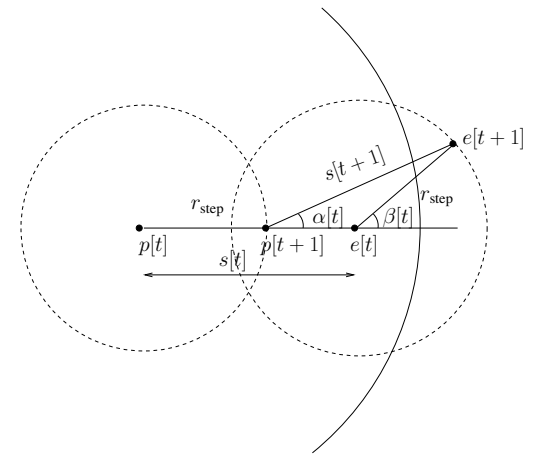

Fig. 2. Relation between angle of deviation and evasion angle

segment $Y Z$ be of length $\frac{r_{\text {step }}}{2}$ and perpendicular to $p\left[t_{0}\right] e\left[t_{0}\right]$ at $X$ and with $X$ as its midpoint. $\angle Y p\left[t_{0}\right] Z$ is called the cone angle. The cone is fixed as long as the evader is in the interior of the cone.

(ii) For $k>0$, let $t_{k}$ denote the time at which the evader steps out of the $(k-1)^{\text {th }}$ cone. Construct $\mathcal{C}_{k}$ analogous to part (i) of this definition by replacing $p\left[t_{0}\right]$ by $p\left[t_{k}\right]$ and $e\left[t_{0}\right]$ by $e\left[t_{k}\right]$. Once the evader leaves the cone, $a$ new cone is constructed which has the same properties as described in part (i).

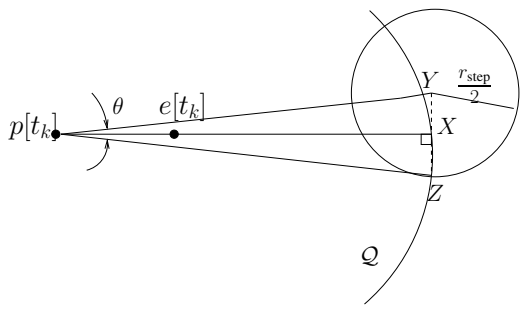

Fig. 3. Construction of cone $\mathcal{C}_{k}$.

The cone described above can be shown to have some useful properties as follows.

Proposition III.7 (Cone properties) (i) There exists a positive angle $\theta$ less than or equal to any cone angle.

(ii) The number of steps $N^{*}$ for which the evader can remain inside the cone without being captured, satisfies

$$
N^{*} \leq\left\lceil\frac{\operatorname{diam}(\mathcal{Q})}{\frac{\sqrt{3}}{2} r_{\text {step }}}\right\rceil .
$$

Proof: (i) The first claim follows from the fact that the region is bounded and hence has a finite diameter, $\operatorname{diam}(\mathcal{Q})$. Thus,

$$
\min _{p[0], e[0] \in \mathcal{Q}} \theta=2 \tan ^{-1}\left(\frac{r_{\text {step }}}{4 \operatorname{diam}(\mathcal{Q})}\right) .
$$

From the construction of the cone, it follows that the motion disc of the pursuer is never tangent to the boundary of a cone. We now compute an upper bound on the number of steps a pursuer would take so that its motion disc passes through every point in the cone. Clearly, this is also an upper bound for the number of steps an evader can remain inside a cone. It 
is evident that the dotted path shown in Figure 4 has greater length than any pursuer path in the cone with maximum step size at each instant and which passes through every point in the cone. The length $L$ of the rectangle is at most equal to $\operatorname{diam}(\mathcal{Q})$. Thus, equation (3) follows from simple geometry.

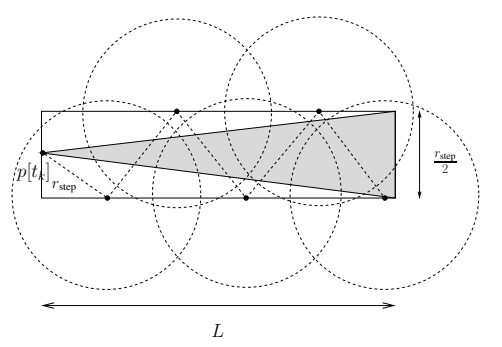

Fig. 4. Upper bound on the number of steps inside a cone for the pursuer

We now state two key results which would be used shortly.

Lemma III.8 (Maximum evasion angle) Let $\beta_{\max }$ denote the maximum value of the evasion angle at the end of each pursuer move. If the pursuer uses the greedy strategy, then

$$
\beta_{\max }=\cos ^{-1}\left(\frac{\left(\kappa^{2}-1\right) r_{\text {step }}^{2}-s^{2}[t]}{2 s[t] r_{\text {step }}}\right),
$$

where $s[t]=\|p[t]-e[t]\|$.

This can be seen by applying the cosine rule to $\triangle p[t] e[t] e[t+$ $1]$, shown in Figure 5. The notation $\triangle A B C$ stands for triangle formed by points $A, B$ and $C$.

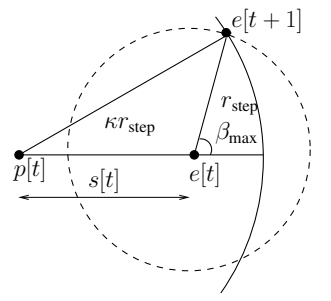

Fig. 5. Constraint on maximum evasion angle

\section{Lemma III.9 (Constraint on maximum evasion angle)}

For the evader to move out of any cone, described in Definition III.6, the maximum evasion angle $\beta_{\max }$ must satisfy,

$$
\left|\beta_{\max }\right|>\frac{\theta}{2 N^{*}}=: \beta_{\mathrm{c}}
$$

where $\theta$ and $N^{*}$ are given in Proposition III.7

Proof: For the evader to step out of cone $\mathcal{C}_{k}$, the sum of the angles of deviation for the pursuer must satisfy,

$$
\sum_{t=t_{k}}^{t_{k+1}}|\alpha[t]|>\frac{\theta}{2}
$$

This is illustrated in Figure 6. From Proposition III.5, we have,

$$
\sum_{t=t_{k}}^{t_{k+1}}|\beta[t]|>\frac{\theta}{2}
$$

Equation (5) now follows from using the bounds on $\theta$ and $N^{*}$ derived in Proposition III.7.

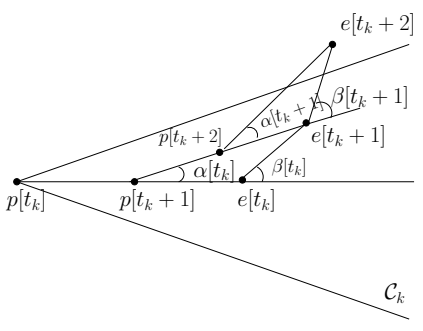

Fig. 6. Illustrating Lemma III.9

We are now ready to prove Theorem III.3.

Proof of Theorem III.3: Two cases need to be considered:

(i) Evader does not move out of a cone: Capture follows from the construction of the cone and from Proposition III.7. Thus, the result is trivially true.

(ii) Evader moves out of a cone: In this case, we seek to show that the evader cannot leave an arbitrarily large number of cones. If the evader steps outside the cone $\mathcal{C}_{k}$, then for some $\tau \in\left\{t_{k}, \ldots, t_{k+1}-1\right\}, \beta[\tau]>\beta_{\mathrm{c}}$. Applying the cosine rule to $\triangle p[\tau] e[\tau] e[\tau+1]$, we obtain,

$$
\begin{aligned}
s^{2}[\tau+1] & =r_{\text {step }}^{2}+\left(s[\tau]-r_{\text {step }}\right)^{2} \\
& +2 r_{\text {step }}\left(s[\tau]-r_{\text {step }}\right) \cos \beta[\tau], \\
\Rightarrow s^{2}[\tau]-s^{2}[\tau+1] & =2 r_{\text {step }}\left(s[\tau]-r_{\text {step }}\right)(1-\cos \beta[\tau]) .
\end{aligned}
$$

Using Equation (5) and since

$$
s[\tau]+s[\tau+1] \leq 2 \kappa r_{\text {step }},
$$

we obtain,

$$
s[\tau+1]-r_{\text {step }} \leq\left(1-\frac{\left(1-\cos \left(\frac{\theta}{2 N^{*}}\right)\right)}{\kappa}\right)\left(s[\tau]-r_{\text {step }}\right) .
$$

Let $\chi_{k}:=s\left[t_{k}\right]-r_{\text {step. }}$. Thus,

$$
\begin{aligned}
& \chi_{k+1} \leq s[\tau+1]-r_{\text {step }}, \\
& \leq\left(1-\frac{\left(1-\cos \left(\frac{\theta}{2 N^{*}}\right)\right)}{\kappa}\right)\left(s[\tau]-r_{\text {step }}\right), \\
& \leq\left(1-\frac{\left(1-\cos \left(\frac{\theta}{2 N^{*}}\right)\right)}{\kappa}\right) \chi_{k},
\end{aligned}
$$

where the first and third inequalities follow from the fact that distance $s[t]$ is non-increasing in the greedy strategy and the second inequality follows from equation (6). Since the term in the parenthesis is strictly less than $1, \chi_{k} \rightarrow 0$ asymptotically, which means that the distance between the pursuer and evader tends to $r_{\text {step }}$ asymptotically. For $\kappa>2$, the distance will reduce to $(\kappa-1) r_{\text {step }}$ after finite time. Thus, the motion disc of the evader is completely contained within the sensing disc of the pursuer. Hence, the result follows. 
The case of $\kappa=2$ : We have seen that the distance $s[t]$ between the pursuer and evader tends asymptotically to $r_{\text {step }}$. From Lemma III.8, we obtain that as $s[t] \rightarrow r_{\text {step }}$, the angle $\beta_{\max } \rightarrow 0$. So, after some finite time,

$$
\beta_{\max }<\frac{\theta}{2 N^{*}}=: \beta_{\mathrm{c}} .
$$

Thus, evader is confined to the current cone according to Lemma III.9 and from Proposition III.7 and we can see from part (i) of this proof, that the pursuer will trap the evader within its sensing radius.

If $\kappa<2$ : There exists a maximum value of the evasion angle at each step, so that the evader's next step is not in the pursuer's present sensing disc. This is shown in Figure 7. The key idea of this part of the proof is that if we ensure that this maximum angle is less than the minimum value needed for the evader to escape a cone, then the evader is forced to remain inside the cone and trapping follows from part (i). The pursuer employs the greedy strategy until the distance

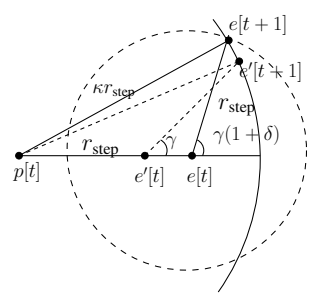

Fig. 7. Illustrating parameters in Equation (8)

is reduced to such a value that the maximum evasion angle is less than or equal to $\gamma(1+\delta)$, where $\gamma$ is the maximum evasion angle if the evader is at $e^{\prime}[t]$, on the stepping radius of the pursuer and $\delta$ is some positive number. At this time instant $t_{\text {final }}$, let the pursuer construct a new cone, $\mathcal{C}_{\text {final }}$. Now if,

$$
\gamma(1+\delta) N^{*}=\min _{p, e \in \mathcal{Q}} \frac{\theta}{2},
$$

where $N^{*}$ and $\theta$ are defined in Proposition III.7, then for some $\tau \in\left\{t_{\text {final }}, \ldots, t_{\text {final }}+N^{*}\right\}, \beta[\tau] \geq \gamma(1+\delta)=\beta_{\mathrm{c}}$ for the evader to leave $\mathcal{C}_{\text {final }}$. This means that the evader is forced to step inside the current sensing radius of the pursuer or remain inside the final cone $\mathcal{C}_{\text {final }}$. In both cases, the pursuer traps the evader within its sensing radius. From Equation (8),

$$
\gamma<\min _{p, e \in \mathcal{Q}} \frac{\theta}{2 N^{*}}=\beta_{\mathrm{c}} .
$$

Applying the cosine rule to $\triangle p[t] e^{\prime}[t] e^{\prime}[t+1]$,

$$
\kappa=\sqrt{2+2 \cos \gamma}>\sqrt{2+2 \cos \beta_{\mathrm{c}}} .
$$

Computing upper bound on time: We have seen that when the pursuer uses the greedy strategy, the evader cannot leave arbitrarily large number of cones. Thus, to compute an upper bound on the trapping time, we compute an upper bound on the number of cones the evader can leave. We have seen that using greedy strategy, $\beta_{\max } \leq \beta_{\mathrm{c}}$ after finite time. From equation (4), we want to determine that distance $s_{\mathrm{c}}$ for which $\beta_{\max }=\beta_{\mathrm{c}}$, so that subsequently, the evader is confined to the same cone. Thus,

$$
s_{\mathrm{c}}=\left(\sqrt{\kappa^{2}-\sin ^{2} \beta_{\mathrm{c}}}-\cos \beta_{\mathrm{c}}\right) r_{\text {step }} .
$$

If $k$ is the final cone index, then using equation (7),

$$
s_{\mathrm{c}}-r_{\text {step }} \leq \chi_{k} \leq \lambda \chi_{\mathrm{k}-1} \leq \cdots \leq \lambda^{k}(\kappa-1) r_{\text {step }},
$$

where $\lambda=1-\frac{1-\cos \left(\frac{\theta}{2 N^{*}}\right)}{\kappa}$ and the worst-case $\chi_{0}=(\kappa-$ 1) $r_{\text {step. }}$ Upon simplifying,

$$
k \leq\left\lceil\frac{\log \left(\frac{\kappa-1}{\sqrt{\kappa^{2}-\sin ^{2} \beta_{\mathrm{c}}}-\cos \beta_{\mathrm{c}}-1}\right)}{\log \frac{1}{\lambda}}\right\rceil .
$$

The result now follows from the fact that for the case of $\kappa<2$, we construct an extra final cone and the maximum number of steps in each cone can be $N^{*}$. The asymptotic result follows by routine simplifications.

We now provide a sufficient condition that ensures evasion against the greedy strategy.

Theorem III.10 (Evasion) Given an environment $\mathcal{Q}$, evasion is possible against the greedy strategy of the pursuer if

(i) $\kappa \notin\left(\sqrt{2+2 \cos \beta_{\mathrm{c}}}, \infty\right)$, where $\beta_{\mathrm{c}}$ is given in Theorem III.3 and

(ii) if there exists a closed curve $\Omega \in \mathcal{Q}$ satisfying,

$$
\rho \geq \frac{r_{\text {step }}}{\sqrt{4-\kappa^{2}}}
$$

where $\rho$ is the radius of curvature at any point on $\Omega$.

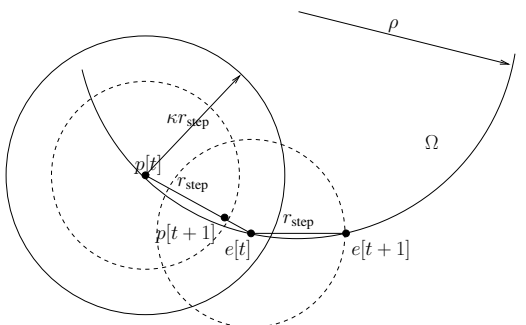

Fig. 8. Evasion using the closed curve $\Omega$

Proof: If equation (9) is satisfied, then there exists a circle $\Omega$ of radius $\rho$ in $\mathcal{Q}$. Suppose the pursuer and the evader are on $\Omega$ as shown in Figure 8 . The evader strategy would be to choose a point $e[t+1]$ on $\Omega$ such that $\| e[t]-e[t+$ $1] \|=r_{\text {step }}$. Since $\rho$ satisfies equation (9), $e[t+1]$ will lie outside the pursuer's sensing disc at time $t$. In the proof of Theorem III.3, we have shown that using the greedy strategy, $s[t] \rightarrow r_{\text {step }}$ asymptotically. Thus, the pursuer approaches $\Omega$ asymptotically. Since $\rho$ satisfies equation (9) and since $\kappa \notin$ $\left(\sqrt{2+2 \cos \beta_{\mathrm{c}}}, \infty\right)$, the evader's new position falls outside pursuer's sensing disc. Thus, using this strategy, the evader can avoid stepping inside the pursuer's current sensing disc indefinitely. 


\section{The Capture phase}

Once the evader is trapped within the sensing range of the pursuer, the pursuer employs the Lion strategy [5] to complete the capture. For the sake of completeness, we now give a brief description of the Lion strategy, adapted to the present problem setting and an upper bound on the time to capture. A similar upper bound for capture time has been obtained by Isler et al. for polygonal environments [12].

In this phase, the new position of the evader is within the current sensing range of the pursuer and hence, $y^{e}[t+1]=$ $e[t+1]$. The Lion strategy is applied to this phase as follows.

(i) At the beginning of the $(t+1)^{t h}$ move of the pursuer, the pursuer constructs the line $e[t] p[t]$, as shown in Figure 9. Let this line intersect the boundary of $\mathcal{Q}$ at $X[t]$ such that $p[t]$ lies between $e[t]$ and $X[t]$.

(ii) The pursuer constructs the line $e[t+1] X[t]$. It moves to the intersection of this line and the circle centered at $p[t]$ and of radius $r_{\text {step }}$. Of the two possible points, the pursuer moves to the point closer to $e[t+1]$.

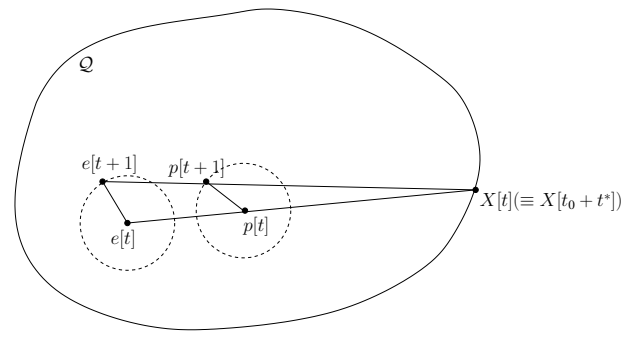

Fig. 9. Using the Lion strategy

A simple analysis reveals that $X[t] \equiv X\left[t_{0}+t^{*}\right]$, for $t \geq$ $\left(t_{0}+t^{*}\right)$, where $\left(t_{0}+t^{*}\right)$ is the time at the end of the pursuit phase. The Lion strategy gives us the following results.

Theorem III.11 (Lion strategy [5]) After trapping the evader within the sensing radius, upon using the Lion strategy by the pursuer,

(i) the distance, $s[t]=\|p[t]-e[t]\|$, is non-increasing after every pursuer move and

(ii) the pursuer captures the evader using the Lion strategy in at most $\left[\frac{\operatorname{diam}^{2}(\mathcal{Q})}{r_{\text {step }}^{2}}\right]$ time steps.

Part (i) of Theorem III.11 ensures that at each instant in the Capture phase, the motion disc of the evader is contained in the sensing disc of pursuer at the end of each pursuer move. Part (ii) completes the capture.

\section{Conclusion And Future Directions}

We have addressed the case of sensing constrained to a closed disc. We proposed a sweep-pursuit-capture pursuer strategy when the evader follows a reactive rabbit model initially. The pursuer detects the evader in its sensing disc and moves toward the last-sensed position of the evader. For a given range of values of the sensing-to-stepping radii $\kappa$ of the players, we established that the evader's motion disc falls completely inside the pursuer's sensing disc in finite time.
We give a novel upper bound for the number of time steps required for this to occur. The final capture is achieved using the established Lion strategy.

A direction for future research would be to look for alternative pursuit strategies that guarantee capture for every bounded convex environment when $\kappa<2$. Also, it is not clear whether there exists any capture strategy which relies solely on the current and possibly next positions of the evader.

\section{ACKNOWLEDGMENTS}

This material is based upon work supported in part by ARO MURI Award W911NF-05-1-0219 and NSF SENSORS Award IIS-0330008 and by the Institute for Collaborative Biotechnologies through the grant DAAD19-03-D-0004 from the U.S. Army Research Office.

\section{REFERENCES}

[1] V. Isler, S. Kannan, and S. Khanna, "Randomized pursuit-evasion with local visibility," SIAM Journal on Discrete Mathematics, vol. 1, no. 20, pp. 26-41, 2006.

[2] Y. Ho, A. E. Bryson, and S. Baron, "Differential games and optimal pursuit-evasion strategies," IEEE Transactions on Automatic Control, vol. 10 , no. 4, pp. 385-389, 1965.

[3] S. Lim, T. Furukawa, G. Dissanayake, and H. F. Durrant-Whyte, "A time-optimal control strategy for pursuit-evasion games problems," in IEEE Int. Conf. on Robotics and Automation, New Orleans, LA, Apr. 2004, pp. 3962-3967.

[4] M. Pachter, "Simple motion pursuit-evasion differential games," in Mediterranean Conference on Control and Automation, Lisbon, Portugal, July 2002, Electronic Proceedings.

[5] J. Sgall, "A solution of David Gale's lion and man problem," Theoretical Computational Science, vol. 259, no. (1-2), pp. 663-670, 2001.

[6] S. Kopparty and C. V. Ravishankar, "A framework for pursuit evasion games in $\mathbb{R}^{n}$," Information Processing Letters, vol. 96, no. 3, pp. $114-122,2005$.

[7] S. Alexander, R. Bishop, and R. Ghrist, "Pursuit and evasion in nonconvex domains of arbitrary dimension," in Robotics: Science and Systems, Philadelphia, PA, Aug. 2006, to appear.

[8] L. Alonso, A. S. Goldstein, and E. M. Reingold, "Lion and Man: Upper and lower bounds," ORSA Journal of Computing, vol. 4, no. 4, pp. 447-452, 1992.

[9] S. M. LaValle and J. Hinrichsen, "Visibility-based pursuit-evasion: The case of curved environments," IEEE Transactions on Robotics and Automation, vol. 17, no. 2, pp. 196-201, 2001.

[10] L. J. Guibas, J.-C. Latombe, S. M. LaValle, D. Lin, and R. Motwani, "Visibility-based pursuit-evasion in a polygonal environment," in $I n$ ternational Workshop on Algorithms and Data Structures, London, UK, 1997, pp. 17-30.

[11] S. Sachs, S. M. LaValle, and S. Rajko, "Visibility-based pursuitevasion in an unknown planar environment," International Journal of Robotics Research, vol. 23, no. 1, pp. 3-26, 2004.

[12] V. Isler, S. Kannan, and S. Khanna, "Randomized pursuit-evasion in a polygonal environment," IEEE Transactions on Robotics, vol. 5, no. 21 , pp. 864-875, 2005.

[13] B. P. Gerkey, S. Thrun, and G. Gordon, "Visibility-based pursuitevasion with limited field of view," in National Conference on Artificial Intelligence, San Jose, CA, July 2004, pp. 20-27.

[14] J. P. Hespanha, H. J. Kim, and S. Sastry, "Multiple-agent probabilistic pursuit-evasion games," Electrical Engineering and Computer Science, University of California at Berkeley, Tech. Rep., Mar. 1999.

[15] R. Vidal, O. Shakernia, H. Kim, D. H. Shim, and S. Sastry, "Probabilistic pursuit-evasion games: Theory, implementation and experimental evaluation," IEEE Transactions on Robotics and Automation, vol. 18, no. 2, pp. 662-669, 2002. 